\title{
Nachhaltigkeitsstrategie auf kommunaler Ebene unter Einbeziehung von Multi-Stakeholder-Diskursen
}

\author{
Kommunen stehen vor zahlreichen Heraus- \\ forderungen zur Förderung einer nachhaltigen \\ Entwicklung. Diese betreffen nicht nur \\ Kommunalpolitik und Verwaltung, sondern auch \\ die Zivilgesellschaft. Wie kann dieser Wandel \\ Richtung Nachhaltigkeit angestoßen und erfolg- \\ reich umgesetzt werden? \\ Von Jessica Scherf und Lutz Becker
}

\section{Einleitung}

Soziale, kulturelle, ökologische Problemfelder sind heute bereits so präsent, dass niemand mehr die Augen vor ihnen verschließen kann. Die öffentliche Kommunikation über Armut, Hungersnöte, aktuelle und zukünftige Extremwetterereignisse oder das unachtsame Verhalten vieler Menschen hat eines verdeutlicht: die unabdingbare Notwendigkeit der Implementierung des Nachhaltigkeitsgedankens in das Handeln und Denken von Individuen, Gesellschaft, Unternehmen, Kommunen und Ländern. Daher sind Akteur/innen auf allen Ebenen der Gesellschaft dazu aufgerufen, sich diesen Problemfeldern entgegenzustellen und damit den Wandel zur nachhaltigen Entwicklung voranzutreiben. Die Ziele für eine nachhaltige Entwicklung wurden von den Vereinten Nationen in Form der 17 Sustainable Development Goals (SGDs) - auch bekannt unter Agenda 2030 - formuliert und am 25. September 2015 verabschiedet. Diese berücksichtigen ökonomische, ökologische wie auch soziale Zielsetzungen, anhand derer Staaten ihre nachhaltige Entwicklung orientieren sollen: unter anderem keine Armut, weniger Ungleichheiten, Maßnahmen zum Klimaschutz, nachhaltige Städte und Gemeinden sowie Partnerschaften zur Erreichung der Ziele (UN 2015). Insbesondere das Ziel der nachhaltigen Städte und Gemeinden steht im Mittelpunkt dieser Betrachtungen. Dazu werden der Prozess der Stadt Solingen in Zusammenarbeit mit der Landesarbeitsgemeinschaft Agenda 21 NRW e. V. (LAG 21 NRW) und Servicestellen „Kommunen in der einen Welt" im Auftrag des Bundesministeriums für wirtschaftliche Zusammenarbeit und Entwicklung be- trachtet, in dem die Stadt Solingen im Rahmen eines Modellprojekts zur Umsetzung der globalen Nachhaltigkeitsziele in NRW-Kommunen ihren Weg zur nachhaltigen Kommune gestaltet (Stadt Solingen 2017a). Dem soll das Wuppertaler Projekt „Aufbruch am Arrenberg e. V.“ gegenübergestellt werden. Auf Grundlage dessen soll folgende Forschungsfrage beantwortet werden: Wie prägen Multi-Stakeholder-Diskurse die Rahmenbedingungen kommunaler Nachhaltigkeitsprojekte?

\section{Ausgewählte Ansätze zur Transformation der Stadt}

Der Sozialpsychologe Kurt Lewin gilt mit seinem klassischen Konzept zu Veränderungsprozessen aus den Vierzigerjahren als einer der Vordenker des organisationalen Wandels (Burnes 2017). Dabei legte Lewin seinen Fokus auf Veränderungen in Gesellschaft und sozialen Gruppen (Lewin 1947). Sein Drei-Phasen-Modell der Veränderung gilt dabei als Pioniertheorie, die viele Wissenschaftler als Orientierungsgrundlage für weitere Modelle und Ansätze des organisationalen Wandels genutzt haben [1].

Spätestens seit dem Aufkommen des Agilitäts-Begriffs, der im Bereich der Softwareentwicklung entstand, jetzt jedoch auf grundlegende Prozesse und Strategien in Organisationen übertragen wird (Brückner et al. 2016), wird der Gedanke der Linearität zunehmend kritisch gesehen. Vielmehr wird im „agile mindset" (Reichel et al. 2015) von kleinen Wiederholungen in Prozessen ausgegangen, um damit auf Unsicherheit und schnelle Veränderungen reagieren zu können (Termer 2016).

In diesem Sinne folgen transformative Prozesse beziehungsweise des geplanten Wandels einem rekursiven Modell, in dem sich Schritte im Veränderungsprozess gegenseitig vor- aber auch rückwärts beeinflussen. Beck formulierte zwölf Prinzipien, die ein "agile mindset“ (Reichel et al. 2015) ausmachen. Dazu gehören unter anderem die Faktoren Vertrauen unter den beteiligten Stakeholdern, Kommunikation und Verpflichtung, eine positive Einstellung gegenüber einem Change sowie die Fähigkeit, in sich selbst organisierenden Teams zu arbeiten. Dadurch, so Beck, werden bestmögliche Ergebnisse erzielt (Beck 2001).

Anders als Kurt Lewin als Vertreter des klassischen topdown orientierten Ansatzes, betrachtet Davide Brocchi die Transformation, die er als „radikalen Wandel der Gesellschaft, 
der Denkweise und der Lebensstile“ (Brocchi 2017d) bezeichnet, aus einer gegensätzlichen Perspektive [2]. Er beschreibt beispielhaft an dem von ihm mit verantworteten Projekt „Tag des guten Lebens" in Köln, wie kommunaler Wandel zur nachhaltigen Entwicklung organisiert und erfolgreich gestaltet werden kann. Dabei stellt er eine entscheidende Frage: „Wollen wir Transformation über uns ergehen lassen [...], oder lieber selbst gestalten?" (Brocchi 2017d). Brocchi betrachtet Top-down-Initiativen zur Förderung der nachhaltigen Entwicklung als gescheitert (Brocchi 2017c), da seiner Ansicht nach ein erfolgreicher gesellschaftlicher Wandel nicht von „Minderheiten für Minderheiten“ (Brocchi 2017d) gestaltet werden kann. Vielmehr soll eine Transformation in Richtung Nachhaltigkeit durch Bottom-up-Prozesse, also von unten nach oben, und "nach menschlichem Maß“" (Brocchi 2017c) gestaltet werden. Harris Tiddens bringt im Zuge dessen den Begriff der Subsidiarität (Zandonella 2009) ein, der besagt, dass eine staatliche Aufgabe zunächst auf kleinstmöglicher Ebene absolviert werden soll [3]. Dieses Prinzip betrachtet Tiddens auch zur Förderung der nachhaltigen Entwicklung als geeignet: „Höhere Ebenen sollen nicht die Arbeit machen, die die Ebenen, die dichter bei der Basis liegen, besser tun können." (Tiddens 2014)

Kommunaler Wandel soll demnach durch die Schließung „unkonventioneller Allianzen“ (Brocchi 2017 c; Brocchi 2017d) und Bündnisse durch Bürger/innen sowie Unternehmen und Institutionen gestaltet werden. Diese sind notwendig, da nicht nur "Systemkorrekturen [...] und technologische Innovationen“ (Brocchi 2015 b; Brocchi 2017c) zur Erreichung einer nachhaltigen Entwicklung ausreichen, sondern vielmehr ein kultureller, sozialer, wirtschaftlicher Wandel und eine Veränderung gesellschaftlicher Strukturen notwendig sind (Grießhammer et al. 2015; Brocchi 2017c). Als Ziele der urbanen Transformation in Richtung Nachhaltigkeit formuliert Brocchi: „Stärkung der Resilienz bzw. der Widerstandsfähigkeit der Stadt und der Region in einer Zeit multipler Krisen. Das Ermöglichen eines guten Lebens für alle Menschen in der Stadt.“ (Brocchi 2015 a; Brocchi 2017c)

Diese Ziele können nach Brocchi durch fünf notwendige Stellschrauben erreicht werden: Der Begriff Gemeingut beschreibt, dass Bürger/innen ihre Stadt als Gut der Allgemeinheit erkennen und mit diesem nachhaltig umgehen. Mit Selbstermächtigung und Selbstmachen beziehungsweise „Selbstverwaltung“ (Tiddens 2014) wird die Möglichkeit der Bürger/ innen umschrieben, eigenständig etwas zu bewirken beziehungsweise umzugestalten. Erreicht wird diese Selbstverwaltung durch das Schaffen unkonventioneller Bündnisse, in denen es gilt, Verantwortung zu übernehmen sowie Vielfalt und Pluralität innerhalb der Allianzen zu fördern. Auch sollte Transformation als Lernprozess betrachtet werden, der zur Fehlerund Selbstreflexion sowie dem „Wissens- und Erfahrungstransfer" dienen soll (Brocchi 2015 b; Brocchi 2017 c; Brocchi 2017d). Zuletzt nennt Brocchi den Faktor Vertrauen, das notwendig ist, um in unkonventionellen Allianzen „eine wirksamere Form der Reduktion von Komplexität“" (Luhmann 2014) zu schaffen und dadurch zu bewirken, dass "gemeinschaftliche Ressourcen“ (Brocchi 2017c) aktiviert werden und eine zielgerichtete Bewegung folgt. Vertrauen steigert das Wohlbefinden der Menschen, den sozialen Zusammenhalt und macht damit die Selbstverwaltung hin zur nachhaltigen Entwicklung möglich (Brocchi 2017c). Der Faktor Vertrauen ist hier erneut in Bezug auf Agilität zu betrachten (Beck 2001). Nichtsdestotrotz müssen auch dieser Bottom-up-Prozess, die darin zu treffenden Entscheidungen, die Finanzierung sowie die Pluralität der Akteur/innen kritisch reflektiert werden.

\section{Untersuchungsdesign und Forschungs- methodik}

Primäres Untersuchungsobjekt ist der Prozess der Stadt Solingen, der im Rahmen des Modellprojekts „Global nachhaltige Kommune in NRW" umgesetzt beziehungsweise angestoßen wurde. Für die Untersuchung wird ein induktives Vorgehen gewählt, mit dem Ziel, auf Basis dieses Praxisbeispiels der Stadt Solingen Handlungsempfehlungen zur Erstellung einer Nachhaltigkeitsstrategie für andere Kommunen und Akteur/innen abzuleiten. Die Autor/innen nehmen in dieser Arbeit die Rolle der teilnehmenden Beobachtenden ein, da sie an zugrunde liegenden Veranstaltungen selbst aktiv mitgearbeitet haben. Um Möglichkeiten und Grenzen herausarbeiten zu können, gilt das Projekt „Klimaquartier Arrenberg“ als Vergleichsprojekt [4].

Die Analyse erfolgt auf Basis folgender Daten:

- Gedächtnisprotokolle: Beobachtungen der Autor/innen als Vertreter der Wissenschaft bei den Treffen der Steuerungsgruppe am 10. Oktober 2017 sowie 27. Februar 2018 sowie bei der zweiten Solinger Nachhaltigkeitskonferenz am 11. September 2017;

I Dokumente zum Projekt Global nachhaltige Kommune Solingen, die öffentlich zugänglich sind;

I Expert/inneninterviews mit Ariane Bischoff (Koordinatorin Projekt Global nachhaltige Kommune Solingen) sowie Jörg Heynkes (Koordinator Projekt Klimaquartier Arrenberg).

Die Interviews sind leitfadengestützt und enthalten offene Fragen, um tatsächliche Möglichkeiten und Grenzen herausfiltern zu können. Zur Auswertung der Expert/inneninterviews wird die qualitative Inhaltsanalyse nach Mayring (2015) verwendet. Die Ergebnisse werden tabellarisch gegenübergestellt [5].

\section{Gegenüberstellung der Projekte und ihrer Prozessstrukturen}

Der Nachhaltigkeitsprozess der Stadt Solingen unter Einbeziehung unterschiedlicher Akteure aus Verwaltung, Politik und Zivilgesellschaft reicht schon in die frühen Jahre des 21. Jahrhunderts zurück. Im Jahr 2006 wurde ein Leitbild konzeptualisiert und mit Strategien, Maßnahmen und Leitprojekten ausgebaut. 2009 und 2013 erfolgten die Überarbeitungen. Dabei wurde jedoch deutlich, dass operative, handlungsleitende Ziele nicht hinreichend Berücksichtigung fanden (Bischoff 
2017). Die Ausschreibung der LAG 21 im Jahr 2015 für das Modellprojekt Global nachhaltige Kommune in NRW stellte für die Stadt Solingen eine Chance in Richtung nachhaltiger Entwicklung dar, für das sich die Stadt bewarb und letztendlich den $\mathrm{Zu}$ schlag dafür erhielt. Es handelt sich somit um ein Projekt auf Initiative der Stadt Solingen, das top-down gesteuert wird (Bischoff 2017). Die Stadt Solingen ist eine der 15 Kommunen, die im Rahmen dieses Modellprojektes eine Strategie zur nachhaltigen Entwicklung erarbeitet und umsetzt. Als Grundlage dienen die bereits beschriebenen 17 globalen Nachhaltigkeitsziele der UN (Stadt Solingen 2017a).

Die Erarbeitung der Nachhaltigkeitsstrategie erfolgte dabei unter Mitarbeit der folgenden Gruppen:

- Koordination: Ariane Bischoff und deren Stellvertreterin sowie Vertreter der LAG 21 NRW;

I Kernteam (KT): verwaltungsseitiges Gremium;

- Steuerungsgruppe (SG): Stadt- und zivilgesellschaftliches Gremium, Wirtschaft und Wissenschaft [6];

- Gesamte Zivilgesellschaft (ZG): wurde in zwei Nachhaltigkeitskonferenzen der Stadt Solingen eingebunden (Bischoff 2017).

Die Bestandsaufnahme erfolgte durch das KT und die LAG 21, die Ergebnisse wurden jedoch mit der SG gemeinsam diskutiert. Für die in der SG verabschiedeten sechs umfassenden und breit gefächerten Themenfelder [7] wurden mit Hilfe des Inputs der Zivilgesellschaft aus der ersten Solinger Nachhaltigkeitskonferenz in Zusammenarbeit von SG und KT Leitlinien mit Grundmotiven und Grundhaltungen formuliert: „Wo wollen wir [...] uns hin entwickeln?“ (Bischoff 2017). Die strategischen, langfristigen Ziele mit Fokus auf das Jahr 2030 wurden ebenfalls in Zusammenarbeit von SG und KT entwickelt. Ähnlich verhielt es sich bei der Entwicklung operativer, handlungsleitender Ziele, die den Fokus auf Zielvorgaben der nächsten maximal fünf Jahre legten (Bischoff 2017). Die Einbeziehung der Zivilgesellschaft erfolgte erneut bei der zweiten Solinger Nachhaltigkeitskonferenz, bei der in Gruppen zu den sechs Themenfeldern konkrete Maßnahmen und Leitprojekte mit besonderer Strahlkraft entwickelt wurden. Die Entwicklung eines Handlungsprogramms aus diesen vorgeschlagenen Maßnahmen und Projekten ist der Status quo des Prozesses (Bischoff 2017). Die Prozessdarstellung verdeutlicht, dass - abgesehen von der Bestandsaufnahme - der Prozess unter Einbeziehung von Multi-Stakeholder-Diskursen erfolgt. Die Stärke der zivilgesellschaftlichen Vertretung variiert je nach Prozessschritt. Gesteuert wird der Prozess von der Stadt Solingen. Die Entscheidungskompetenz für Zwischenentscheidungen liegt bei der SG oder verwaltungsseitig beim KT [8]. Die finale Entscheidung beziehungsweise der Beschluss, welche Projekte umgesetzt und entsprechend mit finanziellen und personellen Ressourcen ausgestattet werden, obliegt der politischen Ebene (Bischoff 2017). Ariane Bischoff erläuterte, dass bewusst darauf verzichtet wurde, Zwischenschritte beschließen zu lassen, da die operativen Ziele stets im Gesamtkontext der Strategie betrachtet werden müssten (Bischoff 2017).
Mit dem Projekt Klimaquartier Arrenberg verfolgt der Verein „Aufbruch am Arrenberg e. V.“ das Ziel, das Quartier Arrenberg bis 2030 so „umzugestalten, dass ein weitestgehend klimaneutrales, klimafreundliches Leben und Wirtschaften möglich wird“ (Heynkes 2017) [9]. Anders als bei der Stadt Solingen, handelt es sich um eine Bottom-up-Initiative der Zivilgesellschaft, die Transformation in den Bereichen Mobilität, Energie und Ernährung zu forcieren [10]. Ein vorgestalteter Prozess ist beim Arrenberg nicht gegeben. Jörg Heynkes, Vorstand des Vereins, spricht hier von „learning by doing“ (Heynkes 2017). In dieses Projekt sind ehrenamtliche, aktive Personen aus dem Verein, Bewohner und Immobilienbesitzer des Quartiers, aber auch externe Engagierte involviert. Auch hier ist also von einem MultiStakeholder-Diskurs zu sprechen. Entscheidungen, die finanzielle Mittel bedingen, werden vom Vorstand des Vereins getroffen. Innerhalb der Arbeitsgruppen treffen die Akteur/innen projektbezogene autonome Entscheidungen (Heynkes 2017).

\section{Darstellung der Möglichkeiten und Grenzen von Multi-Stakeholder-Diskursen}

Beide Initiativen bieten der Zivilgesellschaft und weiteren Akteurinnen- und Akteursgruppen die Möglichkeit, am jeweiligen Projekt mitzuarbeiten und zu partizipieren. Die Intensität der Zusammenarbeit ist beim Klimaquartier insofern stärker, als der Prozess grundsätzlich von der Zivilgesellschaft gestaltet wird (Heynkes 2017). Die Stadt Solingen verzichtet aufgrund fehlender Kapazitäten, aber - durch die kommunale Ausrichtung - auch aufgrund von einer zu umfangreichen zivilgesellschaftlichen Interessensgemeinschaft auf eine prozessumfassende Einbindung [11]. Beide Expert/innen verdeutlichen die Vorteile von Engagement, Ideenvielfalt und Kreativität, die durch die Einbindung der Stakeholder entstehen. Auch die Umsetzungskraft der „Menschen als Treiber“ wird hervorgehoben (Bischoff 2017; Heynkes 2017). Die Stadt Solingen zeigt die innovative, uneingeschränkte Betrachtung der Bürger/innen positiv auf. Sie ermöglicht es Verwaltung und Politik, neue Problemfelder ausfindig zu machen (Bischoff 2017). Die Struktur sowie die finanziellen Mittel durch die Verwaltung der Stadt Solingen werden beim Klimaquartier Arrenberg als Grenzen des eigenen Projektes angegeben (Bischoff 2017; Heynkes 2017) [12]. Eine stärkere Einbindung und Teilhabe der Zivilgesellschaft und die stärkere Integration individueller Themen wird als Kritik am Top-down-Prozess in Solingen angeführt (Bischoff 2017; Sitzung vom 28. 4. 2017). Um die Bearbeitbarkeit und Handhabbarkeit der Projekte sicherzustellen, müssen die Vorschläge durch die Verwaltung gefiltert werden. Das Beharren auf individuellen Schwerpunkten und die Interessensvielfalt erschweren die Konsensfindung und führten in beobachteten Diskussionen zu Konflikten unter den Stakeholdern (Bischoff 2017; Sitzung vom 28. 04. 2017). Ariane Bischoff betont, dass die Geschwindigkeit des Prozesses zwar ausbaufähig sei, dass ein solches Projekt aber bestmöglich erfolgreich umgesetzt werden kann, wenn die Verwaltung als Koordinator und die Zivil- 


\begin{tabular}{|c|c|c|}
\hline Kriterien & Stadt Solingen & Klimaquartier Arrenberg \\
\hline Zielsetzung und Motivation & $\begin{array}{l}\text { I Langjährige Nachhaltigkeitsbemühungen } \\
\text { I Förderung kommunaler nachhaltiger Entwicklung } \\
\text { I Zukunftsorientierte Gesamtausrichtung } \\
\text { I NRW Kommunen als Vorreiter nachhaltiger Entwicklung }\end{array}$ & $\begin{array}{l}\text { I Klimaneutrales, klimafreundliches Leben und Wirtschaften } \\
\text { ermöglichen } \\
\text { I Zukunft der Gattung Mensch sicherstellen } \\
\text { I Transformationsprozess mitgestalten }\end{array}$ \\
\hline Initiatoren & $\begin{array}{l}\text { Stadt Solingen auf Ausschreibung der LAG } 21 \text { NRW } \\
\text { - Top-down }\end{array}$ & $\begin{array}{l}\text { Zivilgesellschaft des Quartiers Arrenberg } \\
\text { • Bottom-up }\end{array}$ \\
\hline Partizipation der Akteur/innen & $\begin{array}{l}\text { Koordination: Ariane Bischoff und Stellvertreterin } \\
\text { (Stadt Solingen) sowie Martin Schön-Chanishvili } \\
\text { (LAG } 21 \text { NRW) } \\
\text { Kernteam: Verwaltungsseitiges Gremium } \\
\text { Steuerungsgruppe: Stadt- und Zivilgesellschaftliches } \\
\text { Gremium, aus Wirtschaft, Wissenschaft, Zivilgesellschaft, } \\
\text { Politik, Verwaltung, Soziales, Kirche etc. } \\
\text { Gesamte Zivilgesellschaft: Eingebunden in Nachhaltigkeits- } \\
\text { konferenzen }\end{array}$ & $\begin{array}{l}\text { I Verein „Aufbruch am Arrenberg e. V.“ } \\
\text { (aktuell ca. } 160 \text { Mitglieder) } \\
\text { - Vorstand } \\
\text { - Aktive Mitglieder (ehrenamtlich) } \\
\text { Dewohner/innen des Quartiers } \\
\text { I Immobilienbesitzer/innen } \\
\text { I Künstler/innen } \\
\text { I Unternehmer/innen } \\
\text { I Externe Engagierte } \\
\text { I Partner, u. a. aus Wissenschaft und großen Wuppertaler } \\
\text { Betrieben }\end{array}$ \\
\hline & $\begin{array}{l}\text { - Einbindung zahlreicher Stakeholder-Gruppen } \\
\text { - Häufigkeit der Einbindung (Kapazitätsfrage) }\end{array}$ & $\begin{array}{l}\text { - Einbindung zahlreicher Stakeholder-Gruppen } \\
\text { - Keine kommunalen sowie politischen Akteur/innen }\end{array}$ \\
\hline Prozessausrichtung & $\begin{array}{l}\text { Top-down } \\
\text { (unter Einbeziehung von Multi-Stakeholder-Diskursen) } \\
\text { I Klassischer Prozess } \\
\text { (angelehnt an St. Gallener Managementsystem) } \\
\text { - Bestandsaufnahme } \\
\text { - Leitlinien } \\
\text { - Strategie } \\
\text { - Operative Ziele } \\
\text { - Maßnahmen } \\
\text { I Vorgehensweise vorgeschlagen von LAC } 21 \text { NRW, } \\
\text { umgesetzt so auch in anderen teilnehmenden Kommunen }\end{array}$ & $\begin{array}{l}\text { Bottom-up } \\
\text { "Ich würde mal sagen, relativ einfach: machen“ } \\
\text { I Keine geplante Vorgehensweise } \\
\text { "learning by doing" }\end{array}$ \\
\hline Entscheidungsträger/innen & $\begin{array}{l}\text { I Finale Beschlüsse: Kommunale Politik } \\
\text { I Zwischenentscheidungen: Steuerungsgruppe und Kernteam } \\
\text { (um weitere Bearbeitung sicherzustellen) } \\
\text { I Vorherige Absicherung durch politische Beschlüsse und } \\
\text { Unterzeichnung von Resolution } \\
\text { I Spiegelung der Zwischenergebnisse in Verwaltungs- } \\
\text { spitze }\end{array}$ & $\begin{array}{l}\text { Entscheidungen über finanzielle Ressourcen: } \\
\text { Vorstand des Vereins } \\
\text { Entscheidungen innerhalb der Arbeitsgruppen werden } \\
\text { dort autonom getroffen }\end{array}$ \\
\hline
\end{tabular}

Tabelle 1: Übersicht der Analyseergebnisse

gesellschaft mit ihren Ideen, Problemfeldern und Engagement zusammenwirken (Bischoff 2017). Jörg Heynkes vom Arrenberg hingegen kritisiert, dass die nachhaltige Langzeitwirkung bei Top-down-Projekten nicht gegeben sei (Heynkes 2017) [13].

Einig sind sich die Expert/innen dahingehend, dass die Größe eines Projektes irrelevant für das gewählte Vorgehen sei. Vielmehr sei es entscheidend, die Menschen und Akteur/ innen als Schlüsselfaktoren frühzeitig zu gewinnen (Bischoff 2017; Heynkes 2017), auch wenn auf kommunaler Ebene eine Einbindung aller Stakeholder nicht möglich ist (Bischoff 2017).

Um den Wandel zur nachhaltigen Entwicklung zukünftig positiv gestalten zu können, halten beide Expert/innen die Einbindung der Interessensgruppen und die Mobilisierung der Akteur/innen für notwendig, um eine gemeinsame Richtung einzuschlagen (Bischoff 2017; Heynkes 2017). Auch die Sensibilisierung für den Veränderungsgedanken und eine gute inhaltliche Durchdringung seien wichtig, so Bischoff (Bischoff 2017). Jörg Heynkes erachtet den Rückhalt durch Verwaltung und Po- litik als notwendig. Darüber hinaus hält er einen Wandel auf politischer Ebene sowie einen verstärkten Blick der Politik auf die Quartiersebene für relevant (Heynkes 2017).

\section{Fazit und Handlungsempfehlungen für Kommunen}

Aus der Gegenüberstellung sowie der vorherigen Auseinandersetzung mit der Literatur konnten folgende Handlungsempfehlungen für Kommunen zur nachhaltigen Entwicklung abgeleitet werden:

- Gestaltung des kommunalen Wandels unter Einbeziehung unterschiedlicher Stakeholdergruppen zur Steigerung von Ideenvielfalt und Engagement zur Umsetzung („unkonventionelle Bündnisse beziehungsweise Allianzen“ [Brocchi 2017c; Brocchi 2017b]).

- Einbinden der Zivilgesellschaft, um die Kommune und das dortige Leben als „Gemeingut“ $\mathrm{zu}$ betrachten und dadurch 


\begin{tabular}{|c|c|c|}
\hline Kriterien & Stadt Solingen & Klimaquartier Arrenberg \\
\hline Vorteile und Möglichkeiten & $\begin{array}{l}\text { I Querschnittsorientierte, innovativ denkende Personen } \\
\text { I Engagement, Kreativität, Ideenvielfalt } \\
\text { I Umsetzungskraft durch Akteur/innen } \\
\text { I Neue Themenfelder, die Politik und Verwaltung noch } \\
\text { nicht bewusst waren } \\
\text { I Innovative, uneingeschränkte, unverstellte Betrachtung } \\
\text { I Aufschließen neuer Problemfelder } \\
\text { I Zusammenwirken von Top-down und Bottom-up } \\
\text { vorteilhaft } \\
\text { I Struktur durch die Verwaltung } \\
\text { I Finanzielle Investitionen durch Verwaltung }\end{array}$ & $\begin{array}{l}\text { Versteht sich als Bühne/Plattform für interne sowie } \\
\text { externe Ideenentwicklung } \\
\text { - Soziales Miteinander fördern } \\
\text { I Engagement und Ideengenerierung fördern } \\
\text { I Gemeinsam die Lebensqualität verbessern } \\
\text { I Nachhaltige Langzeitwirkung von Bottom-up-Projekten } \\
\text { I Menschen als Treiber }\end{array}$ \\
\hline Kritik und Grenzen & $\begin{array}{l}\text { I Wunsch der Zivilgesellschaft nach mehr Teilhabe und } \\
\text { Einbindung } \\
\text { I Geschwindigkeit des Prozesses } \\
\text { I Stärkere Integration individueller Themen gewünscht } \\
\text { I Handhabbarkeit und Bearbeitbarkeit sind sicherzustellen } \\
\text { Blick auf verfügbare Ressourcen } \\
\text { Interessensvielfalt erschwert Konsensfindung } \\
\text { I Umgang mit Konflikten }\end{array}$ & $\begin{array}{l}\text { I Schwierigkeit, in der Dynamik alle Menschen } \\
\text { mitzunehmen } \\
\text { I Chaos durch fehlende Hierarchie } \\
\text { I Professionalisierung } \\
\text { I Geringe finanzielle Ressourcen } \\
\text { I Notwendigkeit von Fördergeldern }\end{array}$ \\
\hline $\begin{array}{l}\text { Generalisierbarkeit in Bezug } \\
\text { auf Größe }\end{array}$ & $\begin{array}{l}\text { I Generalisierbarkeit nicht größenabhängig } \\
\text { I Frühzeitige, projektbezogene Einbindung von Akteur/innen } \\
\text { notwendig } \\
\text { I Einbindung aller Akteurinnen- und Akteursgruppen nicht } \\
\text { möglich } \\
\text { I In Solingen: Leitlinien zur Bürger/innenbeteiligung }\end{array}$ & $\begin{array}{l}\text { I Generalisierbarkeit nicht größenabhängig } \\
\text { Entscheidendes Problem: Menschen zu gewinnen } \\
\text { - Mensch als Schlüsselfaktor }\end{array}$ \\
\hline Bedingungen für Wandel & $\begin{array}{l}\text { I Zusammenwirken von Top-down und Bottom-up } \\
\text { I Frühzeitige Einbindung relevanter Akteur/innen } \\
\text { I Institutionelle Verankerung von Bürgerbeteiligung } \\
\text { I Gute inhaltliche Vorbereitung und Durchdringung } \\
\text { eines Projektes }\end{array}$ & $\begin{array}{l}\text { "Mach es zu deinem Projekt“ } \\
\text { I Menschen mitnehmen } \\
\text { I Zu Projektbeteiligten machen } \\
\text { I Notwendige Akteur/innen mobilisieren } \\
\text { I Verwaltung und Politik als positive Begleiter } \\
\text { I Erfolgsfaktoren immer projektabhängig }\end{array}$ \\
\hline Zukunftsaspekt für Wandel & $\begin{array}{l}\text { I Einbeziehen der Interessensgruppen } \\
\text { I Konfliktpotenziale und Interessenskonflikte meistern } \\
\text { I Sensibilisierung für den Veränderungsgedanken } \\
\text { I Potenzial des Multi-Stakeholder-Diskurses nutzen }\end{array}$ & $\begin{array}{l}\text { I Notwendigkeit vieler Projekte mit Ziel nachhaltiger } \\
\text { Entwicklung } \\
\text { I Politische Veränderungen } \\
\text { I Fokus auf Quartiersebene legen }\end{array}$ \\
\hline
\end{tabular}

Anmerkung: Die Informationen der Stadt Solingen beziehen sich auf folgende Quelle: Bischoff, A. (2017): Interview der Autoren mit Ariane Bischoff vom 06. 10. 2017. Andere Quellen werden entsprechend gekennzeichnet. Die Informationen des Klimaquartiers Arrenberg beziehen sich auf folgende Quelle: Heynkes, J. (2017): Interview der Autoren mit Jörg Heynkes vom 04. 10. 2017.

(Tabelle 1: Fortsetzung)

die aktive Selbstverwaltung zu gestalten. Gestaltung nachhaltiger Entwicklung auf kultureller Ebene (Grießhammer et al. 2015; Brocchi 2017c).

- Vertrauensvoller und offener Dialog bei Kooperation und Zusammenwirken unterschiedlicher Akteurinnen- und Akteursgruppen, um das aktive Mitwirken zu stärken und Konflikte zu vermeiden (Faktor Vertrauen [Luhmann 2014; Beck 2001] und Widerstand [Lewin 1947]).

- Sicherstellen einer tatsächlichen Umsetzung und finanziellen Unterstützung für entwickelte Maßnahmen und Projekte, um die nachhaltige Langzeitwirkung eines Projektes zu garantieren.

- Kommunale Unterstützung für Entwicklung und Umsetzung kleiner Projekte auf Quartiersebene (Subsidiarität [Tiddens 2014]).

I Entwicklung einer Nachhaltigkeitsstrategie als fortwährender Prozess und Lernprozess (Stichwort Agilität und Lernprozess [Brocchi 2015 b; Brocchi 2017c; Brocchi 2017 d]).
Die Gegenüberstellung hat positive sowie negative Aspekte in beiden Prozessen verdeutlicht. Trotz der unterschiedlichen Strukturen haben beide Projekte eines gemeinsam: Sie gestalten die nachhaltige Entwicklung zusammen mit den Personen, die auf kommunaler Ebene tatsächlich davon betroffen sind mit der Zivilgesellschaft. Die Stadt Solingen zeigt, auch durch ihr Engagement zur Entwicklung von Leitlinien zur Bürger/innenbeteiligung, dass sie vom klassischen verwaltungsorientierten Handeln abkehrt und die Bürger/innen, aber auch die Unternehmen und Initiativen, viel stärker in Prozesse einbindet. Die Stadt Solingen könnte gemeinsam mit der Stadt- und Zivilgesellschaft Initiativen und Projekte entwickeln, die von der Bürger/innengesellschaft selbst auf Quartiersebene umgesetzt werden können, um das Konzept der Selbstverwaltung schrittweise verstärkt in das gesellschaftliche Miteinander $\mathrm{zu}$ etablieren und die positive Sensibilisierung zur Veränderung zu unterstützen. Die Ergebnisse der zweiten Nachhaltigkeitskonferenz in Form einer Maßnahmenübersicht (Stadt Solingen 
2017b) zeigen, dass sich in Solingen etwas bewegt - unter anderem durch Multi-Stakeholder-Diskurse. Solingen ist beispielhaft für andere Kommunen und Vorreiter auf dem Weg zur nachhaltigen Entwicklung.

\section{Anmerkungen}

[1] Siehe unter anderem Kotters (1997): Acht-Phasen Modell.

[2] Als Diplom-Sozialwissenschaftler ist er als Forscher, Publizist und Transformationsmanager tätig (Brocchi 2017 a).

[3] Experte für lernende Organisation und studierter Sinologe.

[4] Offizielle Bezeichnung: „Aufbruch am Arrenberg e. V.“. In diesem Paper wird jedoch zur besseren Lesbarkeit vom Klimaquartier oder Quartier Arrenberg gesprochen.

[5] Dafür werden vorab deduktiv entwickelte Kategorien als Vergleichskriterien genutzt. Die inhaltlichen Ergebnisse ergeben sich aus den induktiv gebildeten Kategorien der Inhaltsanalyse.

[6] In der SG sind Akteur/innen aus den Bereichen Verwaltung, Politik, Kirche, Soziales, Wissenschaft, Wirtschaft, Zivilgesellschaft und vielem mehr vertreten und bilden einen Querschnitt aller relevanten Akteurinnenund Akteursgruppen ab.

[7] Gesellschaftliche Teilhabe, Natürliche Ressourcen und Umwelt, Klima und Energie, Mobilität, Arbeit und Wirtschaft, Globale Verantwortung und Eine Welt.

[8] Hier beispielsweise die Aufnahme bestimmter Projekte in das Handlungsprogramm oder die Aufnahme bestimmter Aspekte in die operativen Ziele

[9] Der Arrenberg ist ein Quartier in der Stadt Wuppertal.

[10] Zivilgesellschaft umfasst hier die dort lebenden und arbeitenden Personen sowie Immobilienbesitzer.

[11] Das Klimaquartier Arrenberg agiert auf Quartiersebene und hat somit einen kleineren Umfang an Interessensgruppen.

[12] Das Klimaquartier Arrenberg ist aktuell in einer Phase, in der aufgrund des großen Wachstums und der Projektdynamik Fördergelder zur Professionalisierung benötigt werden.

[13] Seiner Ansicht nach werden Projekte vielfach entwickelt, beim Einstellen finanzieller Ressourcen hingegen oftmals niedergelegt.

\section{Literatur}

Beck, K. (2001): Manifesto for Agile Software Development. www.agilemanifesto.org

Bischoff, A. (2017): Interview der Autoren mit Ariane Bischoff vom 06. 10. 2017. Stadt Solingen.

Brocchi, D. (2015 a): Nachhaltigkeit als kulturelle Herausforderung. In: Steinkellner, V. (Hrsg.): CSR und Kultur. Corporate Cultural Responsibility als Erfolgsfaktor in Ihrem Unternehmen. Heidelberg, Springer Gabler. 41-70.

Brocchi, D. (2015 b): Über die nachhaltige Transformation der Stadt. Der „Tag des guten Lebens: Kölner Sonntag der Nachhaltigkeit“ 2013-2015: ein soziales Experiment. www.davidebrocchi.eu/wp-content/ uploads/2015/11/2015_Ueber-die-nachhaltige-Transformation-der-Stadt1. pdf

Brocchi, D. (2017 a): Davide Brocchi. Vita. www.davidebrocchi.eu/vita/

Brocchi, D. (2017 b): Transition Neighbourhoods. In: Reinermann, J.-L./ Behr, F. (Hrsg.): Die Experimentalstadt. Kreativität und die kulturelle Dimension der Nachhaltigen Entwicklung. Heidelberg, Springer VS. 131-161.

Brocchi, D. (2017 c): Urbane Transformation. Zum Guten Leben in der eigenen Stadt. Bad Homburg, VAS.

Brocchi, D. (2017 d): Urbane Transformation. Das Gute Leben in der Stadt. Ein Impulsvortrag. Konferenz „Querbeet“, 15. 07. 2017, Köln. www.davidebrocchi.eu/wp-content/uploads/2017/09/2017_QuerbeetKiel-Vortrag-Davide-Brocchi.pdf

Brückner, F./Ameln, F. (2016): Agilität. In: Gruppe. Interaktion. Organisation. Zeitschrift für Angewandte Organisationspsychologie 4: 383-386.

Burnes, B. (2017): Kurt Lewin (1890-1947): The Practical Theorist. In: Szabla,
D. B./Pasmore, W./Barnes, M./Gipson, A. N. (Hrsg.): The Palgrave Handbook of Organizational Change Thinkers. New York, Palgrave Macmillan. 749-763.

Dosch, F. et al. (2018): Stadträume mit menschlichem Maß - Boden, Mobilität, Grün. Bergischer Impuls02. Wuppertal Institut für Klima, Umwelt, Energie. Wuppertal. www.spiekerooger-klimagespraeche.de/ sites/default/files/Bergischer_Impuls_02_O.pdf

Grießhammer, R./Brohmann, B. (2015): Wie Transformationen und gesellschaftliche Innovationen gelingen können. Transformationsstrategien und Modells of Change für nachhaltigen gesellschaftlichen Wandel. Dessau-Roßlau, Umweltbundesamt.

Heynkes, J. (2017): Interview der Autoren mit Jörg Heynkes vom 04. 10. 2017. "Aufbruch am Arrenberg e. V.“.

Lewin, K. (1947): Frontiers in Group Dynamics. Concept, Method and Reality in Social Science; Social Equilibria and Social Change. In: Human Relations, 1/1: 5-41.

Luhmann, N. (2014): Vertrauen. Ein Mechanismus der Reduktion sozialer Komplexität, 5. Aufl. Konstanz, UVK.

Mayring, P. (2015): Qualitative Inhaltsanalyse. Grundlagen und Techniken. Weinheim, Beltz.

Reichel, I./Becker, L. (2015): The Agile Mindset and Motivation: An Exploratory Work about the Connection of the Two Constructs. In: Wald, A. et al. (Hrsg.): Advanced Project Management (Vol. 4): Flexibility and Innovative Capacity. GPM Deutsche Gesellschaft für Projektmanagement e.V. 107-128.

Stadt Solingen (2017 a): Global nachhaltige Kommune in NRW. Nachhaltigkeitsstrategie für die Klingenstadt Solingen. www.solingen.de/ C1257EBDo0357318/files/global_nachhaltige_kommune_ kurzbeschreibung_urheber-klingenstadt-solingen.pdf/\$file/global_ nachhaltige_kommune_kurzbeschreibung_urheber-klingenstadt-solingen. pdPopenElement

Stadt Solingen (2017 b): Maßnahmen - Entwürfe für das Handlungsprogramm der Nachhaltigkeitsstrategie der Stadt Solingen. www.solingen. de/C1257EBDo0357318/files/ergebnis-nh-konferenz-projektentwuerfehandlungsprogramm_urheber-klingenstadt-solingen.pdf/\$file/ ergebnis-nh-konferenz-projektentwuerfe-handlungsprogramm_urheberklingenstadt-solingen.pd??OpenElement

Termer, F. (2016): Determinanten der IT-Agilität. Wiesbaden, Springer.

Tiddens, H. (2014): Wurzeln für die lebende Stadt. Wie wir die Eigenverantwortung von Stadtteilen stärken können und warum diese mehr Wertschätzung verdienen. München, oekom.

United Nations (UN) (2015): Sustainable Development Goals. www.un.org/ sustainabledevelopment/sustainable-development-goals/

Wissenschaftlicher Beirat der Bundesregierung Globale Umweltveränderungen (WBGU) (2016): Der Umzug der Menschheit: Die transformative Kraft der Städte. Berlin, WBGU.

Zandonella, B. (2009): Pocket Europa. EU-Begriffe und Länderdaten. Bonn, Bundeszentrale für politische Bildung.

\section{AUTOR/INNEN + KONTAKT}

Jessica Scherf ist Absolventin des Masterstudiengangs "Sustainable Marketing \& Leadership“ an der Hochschule Fresenius Köln

Tel.: +49 157 30923723, E-Mail: jessica.scherf@web.de

Dr. Lutz Becker ist Professor an der Hochschule Fresenius, Wirtschaft und Medien in Köln, Leiter der

Business School und Studiendekan für den Masterstudiengang „Sustainable Marketing \& Leadership“.

Hochschule Fresenius Köln, Fachbereich

Wirtschaft \& Medien, Im Mediapark 4C, 50670 Köln. Tel.: +49 171 5444-387, E-Mail: lutz.becker@hs-fresenius.de
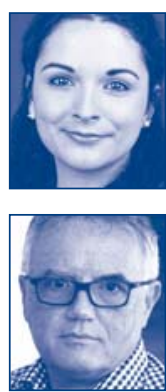